\title{
On future's doorstep: RNA interference and the pharmacopeia of tomorrow
}

\author{
Alan M. Gewirtz \\ Division of Hematology/Oncology, University of Pennsylvania School of Medicine, Philadelphia, Pennsylvania, USA.
}

\begin{abstract}
Small molecules and antibodies have revolutionized the treatment of malignant diseases and appear promising for the treatment of many others. Nonetheless, there are many candidate therapeutic targets that are not amenable to attack by the current generation of targeted therapies, and in a small but growing number of patients, resistance to initially successful treatments evolves. This Review Series on the medicinal promise of posttranscriptional gene silencing with small interfering RNA and other molecules capable of inducing RNA interference (RNAi) is motivated by the hypothesis that effectors of RNAi can be developed into effective drugs for treating malignancies as well as many other types of disease. As this Review Series points out, there is still much to do, but many in the field now hope that the time has finally arrived when "antisense" therapies will finally come of age and fulfill their promise as the magic bullets of the 21 st century.
\end{abstract}

Although the advent of specifically targeted antibodies and small molecules has made an extraordinary difference to the lives of patients with various maladies, many of them suffering from malignant diseases, most of the individuals that physicians are called upon to treat have yet to experience the miracle of targeted therapies. Further, for those that have responded to the various agents available, resistance to once effective remedies is becoming an increasingly important problem (1-3). The importance of the work described in this Review Series on posttranscriptional gene silencing with small interfering RNA (siRNA) derives from the promise of siRNA to simultaneously promote the goal of "targeted, less toxic" therapies and expand the universe of patients who might benefit from them.

Numerous gene silencing strategies have evolved over the years, and these have been primarily directed either to the genes themselves (4-6) or to the mRNAs they encode $(7,8)$. Although some exceptionally clever techniques for direct gene targeting have been developed $(6,9)$, the perceived ease with which mRNA can be accessed has resulted in most therapeutic efforts being directed toward this approach $(10,11)$. A number of modalities are available for mRNA targeting, and of these, the "antisense" strategies have been the most widely applied. These antisense strategies are all based on delivering into cells a nucleic acid strand, either DNA or RNA, that is reverse complementary to the mRNA encoding the protein that one would like to extinguish. By processes still unknown, the antisense nucleic acid (ASNA) strand and the mRNA target come into proximity and then hybridize if the strands are physically accessible to each other. Stable mRNA-ASNA duplexes can interfere with splicing of heteronuclear RNA into mature mRNA $(12,13)$; can block translation of mature mRNA $(14,15)$; or can lead to the destruction of the mRNA, either by endogenous nucleases, such as RNase $H(16,17)$, that are recruited into the mRNA-ASNA duplex or by intrinsic enzymatic activity engineered into the ASNA sequence, as is the case with ribozymes $(18,19)$ and DNAzymes $(20,21)$.

Nonstandard abbreviations used: ASNA, antisense nucleic acid; dsRNA, doublestranded RNA; miRNA, microRNA; RISC, RNA-induced silencing complex; RNAi, RNA interference.

Conflict of interest: The author has declared that no conflict of interest exists. Citation for this article: J. Clin. Invest. 117:3612-3614 (2007). doi:10.1172/JCI34274
In the last several years, RNA interference (RNAi) $(22,23)$ has emerged as an exciting potential alternative to the more classical antisense approaches $(11,24,25)$. Indeed, it is so robust and has had such a profound effect on the way science is now conducted that two of the major contributors to the field were recently awarded a Nobel Prize in Physiology or Medicine for their contributions (26-28). In brief, RNAi is the process by which double-stranded RNA (dsRNA) targets mRNA for destruction in a sequence-dependent manner. The mechanism of RNAi involves processing of dsRNA into approximately 21- to 23-bp fragments that hybridize with the target mRNA and initiate its destruction (Figure 1); this mechanism has been intensively studied. An enzyme called Dicer processes dsRNA into the short fragments (29-32). These small cleavage products are then incorporated into a larger, multi-protein RNA-induced silencing complex (RISC), which simultaneously scans the complementary mRNA sequence for homology to the small, now unwound, RNA fragment and then promotes the destruction of the mRNA through an enzymatic activity integral to the complex $(23,33-35)$. RNAi is in fact a natural process, and this is perhaps best exemplified by the discovery of naturally encoded structural hairpin RNA molecules that are called microRNAs (miRNAs), which are now known to play extremely important roles in regulating gene expression at the posttranscriptional level. Most human miRNA loci are located within intronic regions and are transcribed by RNA polymerase II. The primary transcripts are cleaved by the nuclear ribonuclease Drosha $(36,37)$ to release approximately 70 -nt pre-miRNAs, which are subsequently processed by the RNAi machinery to generate mature, approximately 22 -nt, miRNAs that are increasingly being shown to play a critical role in normal development and malignant cell transformation (38-41).

Although many hope, with good reason, that RNAi will be the "true grail" of targeted therapy, there remain many obstacles that must be overcome before this becomes reality. Indeed, these are quite well known because they are virtually identical to the roadblocks that have plagued the other gene silencing approaches. These issues include the stability of the molecules in plasma and intracellularly $(42,43)$, the ability of these molecules to hybridize with their mRNA target and promote its destruction $(44,45)$, and the ability to deliver these molecules into target cells $(42,46,47)$. 


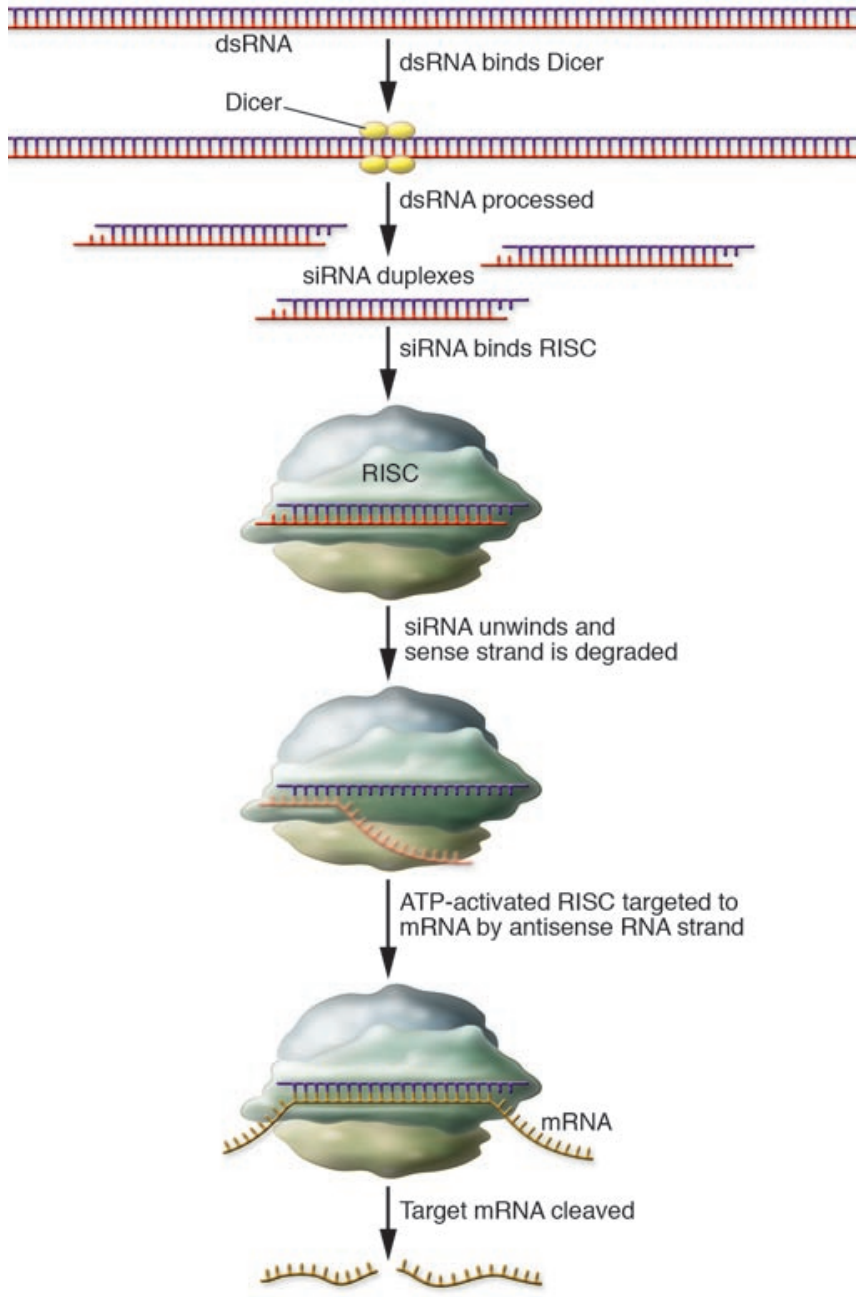

It is also worth noting that unintended silencing with siRNA degradation products (off-target silencing), is also being increasingly recognized as a potential problem, not only as a laboratory artifact but as a source of unwanted medicinal side effects (48-52).

Of these cores issues, stability might seemingly be the most easily tackled, and in the first article of this Review series (53), David Corey discusses how chemically modifying the nucleic acid strand remains an important and active area of research into methods to improve siRNA stability and activity. How to deliver these molecules as a therapeutic remains a major, and largely unsolved, issue, except perhaps for the liver, which seems more amenable to uptake of siRNA molecules than other organs for reasons that are not

\section{Figure 1}

The RNAi pathway. The enzyme Dicer processes dsRNA molecules into shorter dsRNA fragments, typically $21-23$ bp in length, termed siRNA. The siRNA duplexes are incorporated into the RISC, where the sense strand is degraded, leaving the antisense "guide" strand free to hybridize with its complementary sequence in the mRNA. This initiates mRNA strand cleavage by an enzyme native to RISC.

entirely clear. As a general rule, oligodeoxynucleotides are taken up primarily through a combination of adsorptive and fluid-phase endocytosis (54). The mechanism of uptake of dsRNA is likely to be similar and has recently been characterized in liver where it depends on lipoprotein receptors and the mammalian homolog of the C. elegans transmembrane protein $\operatorname{Sid} 1(55,56)$. After internalization, confocal and electron microscopy studies have indicated that the bulk of the oligonucleotides enter the endosome/lysosome compartment, in which most of the material becomes either trapped or degraded. Biological inactivity is the predictable result of these events. In this Review series, Sagir Akhtar and Ibrahim Benter describe the delivery problem and outline possibilities for solving it (57). Sometimes, however, it is necessary to get off the road entirely and just drive around the obstructions that are blocking one's path. If it is not possible to get siRNA molecules into cells, then the strategy of expressing short hairpin, miRNA-like molecules from viral vectors, which can then enter the silencing pathway, might be an approach that could solve the delivery and stability problems simultaneously. This is a classic gene-therapy approach, and its challenges and possibilities are outlined in the Review by Mark Kay and Dirk Grimm (58).

The power of gene silencing with nucleic acid molecules is undisputed in the laboratory, and RNA-based medicines are now working their way into the clinic (59-61). Although some theoretical concerns have already been raised concerning the safety of certain RNAi-mediated therapies $(62,63)$, most workers in this field remain optimistic that the problems described above are solvable and that the dawn of nucleic acid therapeutics, though often promised and now seriously delayed, is finally upon us.

\section{Acknowledgments}

The author would like to acknowledge the support of NIH grants P01 CA72765 and R01 CA101859 and the Leukemia and Lymphoma Society grant 6050-07.

Address correspondence to: Alan M. Gewirtz, Division of Hematology/Oncology, 713 BRB II/III, University of Pennsylvania School of Medicine, 421 Curie Blvd., Philadelphia, Pennsylvania 191046061, USA. Phone: (215) 573-2931; Fax: (215) 573-2078; E-mail: gewirtz@mail.med.upenn.edu.
1. Terui, Y., et al. 1995. Expression of differentiationrelated phenotypes and apoptosis are independently regulated during myeloid cell differentiation. J. Biochem. (Tokyo) 117:77-84.

2. Sawyers, C.L. 2001. Research on resistance to cancer drug Gleevec. Science. 294:1834.

3. Friedberg, J.W. 2005. Unique toxicities and resistance mechanisms associated with monoclonal antibody therapy. Hematology Am. Soc. Hematol. Educ. Program. 2005:329-334.

4. Casey, B.P., and Glazer, P.M. 2001. Gene targeting via triple-helix formation. Prog. Nucleic Acid Res. Mol. Biol. 67:163-192.

5. Urbach, A.R., and Dervan, P.B. 2001. Toward rules for 1:1 polyamide:DNA recognition. Proc. Natl.
Acad. Sci. U. S. A. 98:4343-4348.

6. Durai, S., et al. 2005. Zinc finger nucleases: customdesigned molecular scissors for genome engineering of plant and mammalian cells. Nucleic Acids Res. 33:5978-5990.

7. Gewirtz, A.M., Sokol, D.L., and Ratajczak, M.Z. 1998. Nucleic acid therapeutics: state of the art and future prospects. Blood. 92:712-736.

8. Opalinska, J.B., and Gewirtz, A.M. 2002. Nucleicacid therapeutics: basic principles and recent applications. Nat. Rev. Drug Discov. 1:503-514.

9. Dervan, P.B., and Burli, R.W. 1999. Sequence-specific DNA recognition by polyamides. Curr. Opin. Chem. Biol. 3:688-693.

10. Opalinska, J.B., and Gewirtz, A.M. 2003. Therapeu- tic potential of antisense nucleic acid molecules. Sci. STKE. 2003:pe47.

11. Rossi, J.J. 2006. RNAi therapeutics: SNALPing siRNAs in vivo. Gene Ther. 13:583-584.

12. Kole, R., and Sazani, P. 2001. Antisense effects in the cell nucleus: modification of splicing. Curr. Opin. Mol. Ther. 3:229-234.

13. Dominski, Z., and Kole, R. 1994. Identification and characterization by antisense oligonucleotides of exon and intron sequences required for splicing. Mol. Cell. Biol. 14:7445-7454.

14. Summerton, J., et al. 1997. Morpholino and phosphorothioate antisense oligomers compared in cell-free and in-cell systems. Antisense Nucleic Acid Drug Dev. 7:63-70. 
15. Iversen, P.L. 2001. Phosphorodiamidate morpholino oligomers: favorable properties for sequencespecific gene inactivation. Curr. Opin. Mol. Ther. 3:235-238

16. Zamaratski, E., Pradeepkumar, P.I., and Chattopadhyaya, J. 2001. A critical survey of the structurefunction of the antisense oligo/RNA heteroduplex as substrate for RNase H.J. Biochem. Biophys. Methods. 48:189-208.

17. Crooke, S.T. 1998. Molecular mechanisms of antisense drugs: RNase H. Antisense Nucleic Acid Drug Dev. 8:133-134.

18. Castanotto, D., Scherr, M., and Rossi, J.J. 2000. Intracellular expression and function of antisense catalytic RNAs. Methods Enzymol. 313:401-420.

19. Rossi, J.J. 1999. Ribozymes in the nucleolus. Science. 285:1685.

20. Santoro, S.W., and Joyce, G.F. 1997. A general purpose RNA-cleaving DNA enzyme. Proc. Natl. Acad. Sci. U. S. A. 94:4262-4266.

21. Wu, Y., et al. 1999. Inhibition of bcr-abl oncogene expression by novel deoxyribozymes (DNAzymes). Hum. Gene Ther. 10:2847-2857.

22. Hannon, G.J. 2002. RNA interference. Nature. 418:244-251.

23. Martinez, J., et al. 2002. Single-stranded antisense siRNAs guide target RNA cleavage in RNAi. Cell. 110:563-574.

24. Hexner, E.O., and Gewirtz, A.M. 2005. RNA interference for treating haematological malignancies. Expert Opin. Biol. Ther. 5:1585-1592.

25. Grunweller, A., and Hartmann, R.K. 2005. RNA interference as a gene-specific approach for molecular medicine. Curr. Med. Chem. 12:3143-3161.

26. Fire, A.Z. 2007. Gene silencing by double-stranded RNA (Nobel lecture). Angew Chem. Int. Ed. Engl. 46:6966-6984.

27. Couzin, J. 2006. Nobel Prize in Physiology or Medicine. Method to silence genes earns loud praise. Science. 314:34.

28. Bots, M., Maughan, S., and Nieuwland, J. 2006. RNAi Nobel ignores vital groundwork on plants. Nature. 443:906.

29. Hutvagner, G., et al. 2001. A cellular function for the RNA-interference enzyme Dicer in the maturation of the let-7 small temporal RNA. Science. 293:834-838.

30. Ketting, R.F., et al. 2001. Dicer functions in RNA interference and in synthesis of small RNA involved in developmental timing in C. elegans. Genes Dev. 15:2654-2659.
31. Nicholson, R.H., and Nicholson, A.W. 2002. Molecular characterization of a mouse cDNA encoding Dicer, a ribonuclease III ortholog involved in RNA interference. Mamm. Genome. 13:67-73.

32. Filipowicz, W. 2005. RNAi: the nuts and bolts of the RISC machine. Cell. 122:17-20.

33. Hammond, S.M., Caudy, A.A., and Hannon, G.J. 2001. Post-transcriptional gene silencing by double-stranded RNA. Nat. Rev. Genet. 2:110-119.

34. Parker, J.S., Roe, S.M., and Barford, D. 2004. Crystal structure of a PIWI protein suggests mechanisms for siRNA recognition and slicer activity. $E M B O J$. 23:4727-4737.

35. Rivas, F.V., et al. 2005. Purified Argonaute 2 and an siRNA form recombinant human RISC. Nat. Struct. Mol. Biol. 12:340-349.

36. Han, J., et al. 2004. The Drosha-DGCR8 complex in primary microRNA processing. Genes Dev. 18:3016-3027.

37. Carmell, M.A., and Hannon, G.J. 2004. RNase III enzymes and the initiation of gene silencing. Nat. Struct. Mol. Biol. 11:214-218.

38. Pasquinelli, A.E. 2002. MicroRNAs: deviants no longer. Trends Genet. 18:171-173.

39. Chen, C.Z., et al. 2004. MicroRNAs modulate hematopoietic lineage differentiation. Science. 303:83-86.

40. Calin, G.A., and Croce, C.M. 2006. Genomics of chronic lymphocytic leukemia microRNAs as new players with clinical significance. Semin. Oncol. 33:167-173.

41. Kim, Y.K., and Kim, V.N. 2007. Processing of intronic microRNAs. EMBO J. 26:775-783.

42. Cejka, D., Losert, D., and Wacheck, V. 2006. Short interfering RNA (siRNA): tool or therapeutic? Clin. Sci. (Lond.) 110:47-58.

43. Liao, H., and Wang, J.H. 2005. Biomembrane-permeable and Ribonuclease-resistant siRNA with enhanced activity. Oligonucleotides. 15:196-205.

44. Brown, K.M., Chu, C.Y., and Rana, T.M. 2005. Target accessibility dictates the potency of human RISC. Nat. Struct. Mol. Biol. 12:469-470.

45. Patzel, V., et al. 2005. Design of siRNAs producing unstructured guide-RNAs results in improved RNA interference efficiency. Nat. Biotechnol. 23:1440-1444.

46. Spagnou, S., Miller, A.D., and Keller, M. 2004. Lipidic carriers of siRNA: differences in the formulation, cellular uptake, and delivery with plasmid DNA. Biochemistry. 43:13348-13356.
47. Sioud, M. 2005. siRNA delivery in vivo. Methods Mol. Biol. 309:237-249.

48. Lassus, P., Rodriguez, J., and Lazebnik, Y. 2002. Confirming specificity of RNAi in mammalian cells. Sci. STKE. 2002:PL13.

49. Hornung, V., et al. 2005. Sequence-specific potent induction of IFN-alpha by short interfering RNA in plasmacytoid dendritic cells through TLR7. Nat. Med. 11:263-270.

50. Zhang, Z., et al. 2006. siRNA binding proteins of microglial cells: PKR is an unanticipated ligand. J. Cell. Biochem. 97:1217-1229.

51. Zhang, Y.C., et al. 2005. Antisense inhibition: oligonucleotides, ribozymes, and siRNAs. Methods Mol. Med. 106:11-34.

52. Bitko, V., et al. 2005. Inhibition of respiratory viruses by nasally administered siRNA. Nat. Med. 11:50-55.

53. Corey, D.R. 2007. Chemical modification: the key to clinical application of RNA interference? J. Clin. Invest. 117:3615-3622.

54. Beltinger, C., et al. 1995. Binding, uptake, and intracellular trafficking of phosphorothioatemodified oligodeoxynucleotides. J. Clin. Invest. 95:1814-1823.

55. Hunter, C.P., et al. 2006. Systemic RNAi in Caenorhabditis elegans. Cold Spring Harb. Symp. Quant. Biol. 71:95-100.

56. Wolfrum, C., et al. 2007. Mechanisms and optimization of in vivo delivery of lipophilic siRNAs. Nat. Biotechnol. 25:1149-1157.

57. Akhtar, S., and Benter, I.F. 2007. Nonviral delivery of synthetic siRNAs in vivo. J. Clin. Invest. 117:3623-3632.

58. Grimm, D., and Kay, M.A. 2007. Therapeutic application of RNAi: is mRNA targeting finally ready for prime time? J. Clin. Invest. 117:3633-3641.

59. Fattal, E., and Bochot, A. 2006. Ocular delivery of nucleic acids: antisense oligonucleotides, aptamers and siRNA. Adv. Drug Deliv. Rev. 58:1203-1223.

60. Hadj-Slimane, R., et al. 2007. Short interfering RNA (siRNA), a novel therapeutic tool acting on angiogenesis. Biochimie. 89:1234-1244.

61. de Fougerolles, A., et al. 2007. Interfering with disease: a progress report on siRNA-based therapeutics. Nat. Rev. Drug Discov. 6:443-453.

62. Frantz, S. 2006. Safety concerns raised over RNA interference. Nat. Rev. Drug Discov. 5:528-529.

63. Grimm, D., et al. 2006. Fatality in mice due to oversaturation of cellular microRNA/short hairpin RNA pathways. Nature. 441:537-541. 